\title{
Generalized Krein Parameters of a Strongly Regular Graph
}

\author{
Luís Almeida Vieira1, Vasco Moço Mano² \\ ${ }^{1}$ CMUP-Center of Research of Mathematics of University of Porto, Department of Civil Engineering, University \\ of Porto, Porto, Portugal \\ ${ }^{2}$ CIDMA-Center for Research and Development in Mathematics and Applications, Department of \\ Mathematics, University of Aveiro, Aveiro, Portugal \\ Email: Ivieira@fe.up.pt, vascomocomano@gmail.com
}

Received 11 November 2014; revised 8 December 2014; accepted 22 December 2014

Copyright (C) 2015 by authors and Scientific Research Publishing Inc.

This work is licensed under the Creative Commons Attribution International License (CC BY).

http://creativecommons.org/licenses/by/4.0/

(c) (i) Open Access

\begin{abstract}
We consider the real three-dimensional Euclidean Jordan algebra associated to a strongly regular graph. Then, the Krein parameters of a strongly regular graph are generalized and some generalized Krein admissibility conditions are deduced. Furthermore, we establish some relations between the classical Krein parameters and the generalized Krein parameters.
\end{abstract}

\section{Keywords} Algebraic Combinatorics, Association Schemes, Strongly Regular Graphs, Graphs and Linear
Algebra

\section{Introduction}

In this paper we explore the close and interesting relationship of a three-dimensional Euclidean Jordan algebra $\mathcal{V}$ to the adjacency matrix of a strongly regular graph $X$. According to [1], the Jordan algebras were formally introduced in 1934 by Pascual Jordan, John von Neumann and Eugene Wigner in [2]. There, the authors attempted to deduce some of the Hermitian matrix properties and they came across a structure lately called a Jordan algebra. Euclidean Jordan algebras were born by adding an inner product with a certain property to a Jordan algebra. It is remarkable that Euclidean Jordan algebras turned out to have such a wide range of applications. For instance, we may cite the application of this theory to statistics [3], interior point methods [4] [5] and combinatorics [6]. More detailed literature on Euclidean Jordan algebras can be found in Koecher's lecture notes [7] and in the monograph by Faraut and Korányi [8].

Along this paper, we consider only simple graphs, i.e., graphs without loops and parallel edges, herein called 
graphs. Considering a graph $X$, we denote its vertex set by $V(X)$ and its edge set by $E(X)$-an edge whose endpoints are the vertices $x$ and $y$ is denoted by $x y$. In such case, the vertices $x$ and $y$ are adjacent or neighbors. The number of vertices of $X,|V(X)|$, is called the order of $X$.

A graph in which all pairs of vertices are adjacent (non-adjacent) is called a complete (null) graph. The number of neighbors of a vertex $v$ in $V(X)$ is called the degree of $v$. If all vertices of a graph $X$ have degree $k$, for some natural number $k$, then $X$ is $k$-regular.

We associate to $X$ an $n$ by $n$ matrix $A=\left[a_{i j}\right]$, where each $a_{i j}=1$, if $v_{i} v_{j} \in E(X)$, otherwise $a_{i j}=0$, called the adjacency matrix of $X$. The eigenvalues of $A$ are simply called the eigenvalues of $X$.

A non-null and not complete graph $X$ is $(n, k, a, c)$-strongly regular; if it is $k$-regular, each pair of adjacent vertices has $a$ common neighbors and each pair of non-adjacent vertices has $c$ common neighbors. The parameters of a $(n, k, a, c)$-strongly regular graph are not independent and are related by the equality

$$
k(k-a-1)=(n-k-1) c .
$$

It is also well known (see, for instance, [9]) that the eigenvalues of a $(n, k, a, c)$-strongly regular graph $X$ are $k, \theta$ and $\tau$, where $\theta$ and $\tau$ are given by

$$
\begin{aligned}
& \theta=\left(a-c+\sqrt{(a-c)^{2}+4(k-c)}\right) / 2, \\
& \tau=\left(a-c-\sqrt{(a-c)^{2}+4(k-c)}\right) / 2 .
\end{aligned}
$$

Therefore, the usually called restricted eigenvalues $\theta$ and $\tau$ are such that the former is positive and the latter is negative. Their multiplicities can be obtained as follows (see, for instance, [10]):

$$
\begin{aligned}
& \mu_{1}=\frac{1}{2}\left(n-1-\frac{(\theta+\tau)(n-1)+2 k}{\theta-\tau}\right), \\
& \mu_{2}=\frac{1}{2}\left(n-1+\frac{(\theta+\tau)(n-1)+2 k}{\theta-\tau}\right) .
\end{aligned}
$$

Taking into account the above eigenvalues and their multiplicities, the following additional conditions are widely used as feasible conditions for parameters sets $(n, k, a, c)$ of strongly regular graphs; that is, if $(n, k, a, c)$ is a parameter set of a strongly regular graph, then the equality (1) and each one of the following inequalities holds:

- The nontrivial Krein conditions obtained in [11]:

$$
\begin{aligned}
& (\theta+1)(k+\theta+2 \theta \tau) \leq(k+\theta)(\tau+1)^{2}, \\
& (\tau+1)(k+\tau+2 \theta \tau) \leq(k+\tau)(\theta+1)^{2} .
\end{aligned}
$$

- The Seidel’s absolute bounds qre (see [12]):

$$
n \leq \frac{\mu_{1}\left(\mu_{1}+3\right)}{2} \text { and } n \leq \frac{\mu_{2}\left(\mu_{2}+3\right)}{2} .
$$

With these conditions, many of the parameter sets are discarded as possible parameters sets of strongly regular graphs. To decide whether a set of parameters is the parameter set of a strongly regular graph is one of the main problems on the study of strongly regular graphs. It is worth noticing that these Krein conditions and the Seidel's absolute bounds are special cases of general inequalities obtained for association schemes.

An association scheme with $d$ classes is a finite set $S$ together with $d+1$ relations $R_{i}$ defined on $S$ satisfying the following conditions.

1) The set of relations $\left\{R_{0}, R_{1}, \cdots, R_{d}\right\}$ is a partition of the Cartesian product of $S \times S$.

2) $R_{0}=\{(x, x): x \in S\}$.

3) If $(x, y) \in R_{i}$, then also $(y, x) \in R_{i}, \forall x, y \in S$ and for $i=0, \cdots, d$. 
4) For each $(x, y) \in R_{k}$, the number $p_{i j}^{k}$ of elements $z \in S$ such that $(x, z) \in R_{i}$ and $(z, y) \in R_{j}$ depends only from $i, j$ and $k$.

The numbers $p_{i j}^{k}$ are called the intersection numbers of the association scheme. Some authors call this type of association schemes symmetric association schemes. The relations $R_{i}$ of the association scheme can be represented by their adjacency matrices $A_{i}$ of order $n=|S|$ defined by $\left(A_{i}\right)_{x y}=\left\{\begin{array}{ll}1, & \text { if }(x, y) \in R_{i}, \\ 0, & \text { otherwise. }\end{array}\right.$ We may say that $A_{i}$ is the adjacency matrix of the graph $G_{i}$, with $V\left(G_{i}\right)=S$ and $E\left(G_{i}\right)=R_{i}$. The Bose-Mesner algebra of the association scheme (introduced in [13]) is defined, using these matrices, by the following conditions, which are equivalent to the conditions 1) - 4) of the association scheme:

1) $\sum_{i=0}^{d} A_{i}=J_{n}$,

2) $A_{0}=I_{n}$,

3) $A_{i}=A_{i}^{\mathrm{T}}, \quad \forall i \in\{0, \cdots, d\}$,

4) $A_{i} A_{j}=\sum_{k=0}^{d} p_{i j}^{k} A_{k}, \quad \forall i, j \in\{0, \cdots, d\}$,

where $J_{n}$ is the matrix of order $n$ whose entries are equal to one and $I_{n}$ is the identity matrix of order $n$. From 1) we may conclude that the matrices $A_{i}$ are linearly independent, and from 2) - 4) it follows that they generate a commutative $(d+1)$-dimensional algebra $\mathcal{A}$ of symmetric matrices with constant diagonal. The matrices $A_{i}$ commute and then, they can be diagonalized simultaneously, i.e., there exists a matrix $B$ such that $\forall A \in \mathcal{A}, B^{-1} A B$ is a diagonal matrix. Thus, the algebra $\mathcal{A}$ is semisimple and has a unique complete system of orthogonal idempotents $E_{0}, \cdots, E_{d}$. Therefore, $\sum_{i=0}^{d} E_{i}=I_{n}$ and $E_{i} E_{j}=\delta_{i j} E_{i}$, where $\delta_{i j}= \begin{cases}1, & \text { if } i=j, \\ 0, & \text { otherwise. }\end{cases}$

This paper is organized as follows. In Section 2, a short introduction on Euclidean Jordan algebras with the fundamental concepts is presented. In order to obtain new feasible conditions for the existence of a strongly regular graph, in Section 3, we define the generalized Krein parameters of a strongly regular graph. In Section 4, we establish some relations between the Krein parameters and the generalized Krein parameters, and present some properties of the generalized Krein parameters. Finally, since the generalized Krein parameters are nonnegative we establish new admissibility conditions, for the parameters of a strongly regular graph that give different information from that given by the Krein conditions 6) - 7).

\section{Euclidean Jordan Algebras and Strongly Regular Graphs}

In this section the main concepts of Euclidean Jordan Algebras that can be seen for instance in [8], are shortly surveyed.

Let $\mathcal{V}$ be a real vector space with finite dimension and a bilinear mapping $(u, v) \mapsto u \circ v$ from $\mathcal{V} \times \mathcal{V}$ to $\mathcal{V}$, that satisfies $(u \circ u) \circ u=u \circ(u \circ u), \forall u \in \mathcal{V}$. Then, $\mathcal{V}$ is called a real power associative algebra. If $\mathcal{V}$ contains an element, $e$, such that for all $u$ in $\mathcal{V}, e \circ u=u \circ e=u$, then $e$ is called the unit element of $\mathcal{V}$. Considering a bilinear mapping $(u, v) \mapsto u \circ v$, if for all $u$ and $v$ in $\mathcal{V}$ we have $\left.\left(J_{1}\right)\right] u \circ v=v \circ u$ and $\left(J_{2}\right)$ $u \circ\left(u^{2} \circ v\right)=u^{2} \circ(u \circ v)$, with $u^{2}=u \circ u$, then $\mathcal{V}$ is called a Jordan algebra. If $\mathcal{V}$ is a Jordan algebra with unit element, then $\mathcal{V}$ is power associative (cf. [8]). Given a Jordan algebra $\mathcal{V}$ with unit element $e$, if there is an inner product $\langle\cdot \cdot \cdot\rangle$ that verifies the equality $\langle u \circ v, w\rangle=\langle v, u \circ w\rangle$, for any $u, v, w$ in $\mathcal{V}$, then $\mathcal{V}$ is called an Euclidean Jordan algebra. An element $c$ in an Euclidean Jordan algebra $\mathcal{V}$, with unit element $e$, is an idempotent if $c^{2}=c$. Two idempotents $c$ and $d$ are orthogonal if $c \circ d=0$. We call the set $\left\{c_{1}, c_{2}, \cdots, c_{k}\right\}$ a complete system of orthogonal idempotents if (i) $c_{i}^{2}=c_{i}, \forall i \in\{1, \cdots, k\}$; (ii) $c_{i} \circ c_{j}=0$, $\forall i \neq j$ and (iii) $c_{1}+c_{2}+\cdots+c_{k}=e$.

Let $\mathcal{V}$ be an Euclidean Jordan algebra with unit element $e$. Then, for every $u$ in $\mathcal{V}$, there are unique distinct real numbers $\lambda_{1}, \lambda_{2}, \cdots, \lambda_{k}$, and an unique complete system of orthogonal idempotents $\left\{c_{1}, c_{2}, \cdots, c_{k}\right\}$ such that

$$
u=\lambda_{1} c_{1}+\lambda_{2} c_{2}+\cdots+\lambda_{k} c_{k}
$$

with $c_{j} \in \mathbb{R}[u], \quad j=1, \cdots, k$ (see [8], Theorem III 1.1). These $\lambda_{j}$ 's are the eigenvalues of $u$ and (9) is called the first spectral decomposition of $u$.

The rank of an element $u$ in $\mathcal{V}$ is the least natural number $k$, such that the set $\left\{e, u, \cdots, u^{k}\right\}$ is linear dependent (where $u^{k}=u \circ u^{k-1}$ ), and we write $\operatorname{rank}(u)=k$. This concept is expanded by defining the rank of the 
algebra $\mathcal{V}$ as the natural number $\operatorname{rank}(\mathcal{V})=\max \{\operatorname{rank}(u): u \in \mathcal{V}\}$. The elements of $\mathcal{V}$ with rank equal to the rank of $\mathcal{V}$ are the regular elements of $\mathcal{V}$. This set of regular elements is open and dense in $\mathcal{V}$. If $u$ is a regular element of $\mathcal{V}$, with $r=\operatorname{rank}(u)$, then the set $\left\{e, u, u^{2}, \cdots, u^{r}\right\}$ is linearly dependent and the set $\left\{e, u, u^{2}, \cdots, u^{r-1}\right\}$ is linearly independent. Thus we may conclude that there exist unique real numbers $a_{1}(u), \cdots, a_{r}(u)$, such that $u^{r}-a_{1}(u) u^{r-1}+\cdots+(-1)^{r} a_{r}(u) e=0$, where 0 is the null vector of $\mathcal{V}$. Therefore, with the necessary adjustments, we obtain the following polynomial in $\lambda: p(u, \lambda)=\lambda^{r}-a_{1}(u) \lambda^{r-1}+\cdots+(-1)^{r} a_{r}(u)$. This polynomial is called the characteristic polynomial of $u$, where each coefficient $a_{i}$ is a homogeneous polynomial of degree $i$ in the coordinates of $u$ in a fixed basis of $\mathcal{V}$. Although we defined the characteristic polynomial for a regular element of $\mathcal{V}$, we can extend this definition to all the elements in $\mathcal{V}$, because each polynomial $a_{i}$ is homogeneous and, as above referred, the set of regular elements of $\mathcal{V}$ is dense in $\mathcal{V}$. The roots of the characteristic polynomial of $u, \lambda_{1}, \lambda_{2}, \cdots, \lambda_{r}$ are called the eigenvalues of $u$. Furthermore, the coefficients $a_{1}(u)$ and $a_{r}(u)$ in the characteristic polynomial of $u$, are called the trace and the determinant of $u$, respectively.

From now on, we consider the Euclidean Jordan algebra of real symmetric matrices of order $n, \mathcal{V}$, such that $\forall A, B \in \mathcal{V}, A \circ B=(A B+B A) / 2$, where $A B$ is the usual product of matrices. Furthermore, the inner product of $\mathcal{V}$ is defined as $\langle A, B\rangle=\operatorname{tr}(A B)$, where $\operatorname{tr}$ is the classical trace of matrices, that is the sum of its eigenvalues.

Let $X$ be a $(n, k, a, c)$-strongly regular graph such that $0<c<k<n-1$, and let $A$ be the adjacency matrix of $X$. Then $A$ has three distinct eigenvalues, namely the degree of regularity $k$, and the restricted eigenvalues $\theta$ and $\tau$, given in (2) and (3). Now we consider the Euclidean Jordan subalgebra of $\mathcal{V}, \mathcal{V}^{\prime}$, spanned by the identity matrix of order $n, I_{n}$, and the powers of $A$. Since $A$ has three distinct eigenvalues, then $\mathcal{V}^{\prime}$ is a three dimensional Euclidean Jordan algebra with $\operatorname{rank}\left(\mathcal{V}^{\prime}\right)=3$ and $B=\left\{I_{n}, A, A^{2}\right\}$ is a basis of $\mathcal{V}^{\prime}$.

Let $S=\left\{E_{0}, E_{1}, E_{2}\right\}$ be the unique complete system of orthogonal idempotents of $\mathcal{V}^{\prime}$ associated to $A$. Then

$$
\begin{aligned}
& E_{0}=\frac{A^{2}-(\theta+\tau) A+\theta \tau I_{n}}{(k-\theta)(k-\tau)}=\frac{J_{n}}{n}, \\
& E_{1}=\frac{A^{2}-(k+\tau) A+k \tau I_{n}}{(\theta-\tau)(\theta-k)}, \\
& E_{2}=\frac{A^{2}-(k+\theta) A+k \theta I_{n}}{(\tau-\theta)(\tau-k)},
\end{aligned}
$$

where $J_{n}$ is the matrix whose entries are all equal to 1 . Since $\mathcal{V}^{\prime}$ is an Euclidean Jordan algebra that is closed for the Hadamard product of matrices, denoted by $\bullet$ and $S$ is a basis of $\mathcal{V}^{\prime}$, then there exist real numbers $q_{\alpha 2}^{p}$ and $q_{\alpha \beta 11}^{p}, 1 \leq \alpha, \beta \leq 3, \alpha \neq \beta$, such that

$$
E_{\alpha} \cdot E_{\alpha}=\sum_{p=0}^{2} q_{\alpha 2}^{p} E_{p}, \quad E_{\alpha} \cdot E_{\beta}=\sum_{p=0}^{2} q_{\alpha \beta 11}^{p} E_{p} .
$$

The real numbers, defined in (11), (whose notation will be clarified later) $q_{\alpha 2}^{p}$ and $q_{\alpha \beta 11}^{p}, 1 \leq \alpha, \beta \leq 3$, $\alpha \neq \beta$, are called the "classical" Krein parameters of the graph $X$ (cf. [10]). Since $q_{12}^{1} \geq 0$ and $q_{22}^{2} \geq 0$, the "classical" Krein admissibility conditions $\theta \tau^{2}-2 \theta^{2} \tau-\theta^{2}-k \theta+k \tau^{2}+2 k \tau \geq 0$, and

$\theta^{2} \tau-2 \theta \tau^{2}-\tau^{2}-k \tau+k \theta^{2}+2 k \theta \geq 0$ (presented in [9], Theorem 21.3) can be deduced.

\section{A Generalization of the Krein Parameters}

Herein the generalized Krein parameters of a $(n, k, a, c)$-strongly regular graph are defined and then, necessary conditions for the existence of a $(n, k, a, c)$-strongly regular graph are deduced. These conditions are generalizations of the Krein conditions (see Theorem 21.3 in [9]). Throughout this paper we use a slight different notation from classical books like [9] [14], because, in this way, the connections between the "classical" and the generalized parameters are better understood. Now we generalize the Krein parameters in order to obtain new generalized admissibility conditions on the parameters of strongly regular graphs. Firstly, considering $S=\left\{E_{0}, E_{1}, E_{2}\right\}$ defined like in (10) in the Basis $B$, and rewriting the idempotents under the new basis $\left\{I_{n}, A, J_{n}-A-I_{n}\right\}$ of 
$\mathcal{V}^{\prime}$ we obtain

$$
\begin{aligned}
& E_{0}=\frac{\theta-\tau}{n(\theta-\tau)} I_{n}+\frac{\theta-\tau}{n(\theta-\tau)} A+\frac{\theta-\tau}{n(\theta-\tau)}\left(J_{n}-A-I_{n}\right), \\
& E_{1}=\frac{|\tau| n+\tau-k}{n(\theta-\tau)} I_{n}+\frac{n+\tau-k}{n(\theta-\tau)} A+\frac{\tau-k}{n(\theta-\tau)}\left(J_{n}-A-I_{n}\right), \\
& E_{2}=\frac{\theta n+k-\theta}{n(\theta-\tau)} I_{n}+\frac{-n+k-\theta}{n(\theta-\tau)} A+\frac{k-\theta}{n(\theta-\tau)}\left(J_{n}-A-I_{n}\right) .
\end{aligned}
$$

Consider the natural number $p$ and denote by $M_{n}(\mathbb{R})$ the set of square matrices of order $n$ with real entries. Then for $B \in M_{n}(\mathbb{R})$, we denote by $B^{\bullet p}$ and $B^{\otimes p}$ the Hadamard power of order $p$ of $B$ and the Kronecker power of order $p$ of $B$, respectively, with $B^{\cdot 1}=B$ and $B^{\otimes 1}=B$.

Now, we introduce the following compact notation for the Hadamard and the Kronecker powers of the elements of $S$. Let $x, y, z, \alpha, \beta$ and $\gamma$ be natural numbers such that $1 \leq \alpha, \beta, \gamma \leq 3, x \geq 2$ and $\alpha<\beta$. Then we define

$$
\begin{array}{rll}
E_{\alpha}^{\cdot x}=\left(E_{\alpha}\right)^{\cdot x} & \text { and } & E_{\alpha}^{\otimes x}=\left(E_{\alpha}\right)^{\otimes x}, \\
E_{\alpha \beta}^{\cdot y z}=\left(E_{\alpha}\right)^{\cdot y} \cdot\left(E_{\beta}\right)^{\cdot z} & \text { and } & E_{\alpha \beta}^{\otimes y z}=\left(E_{\alpha}\right)^{\otimes y} \otimes\left(E_{\beta}\right)^{\otimes z},
\end{array}
$$

Again, since the Euclidean Jordan algebra $\mathcal{V}^{\prime}$ is closed under the Hadamard product and $S$ is a basis of $\mathcal{V}^{\prime}$, then there exist real numbers $q_{\alpha x}^{i}, \quad q_{\alpha \beta y z}^{i}, q_{(\alpha \oplus \beta) x}^{i}$ and $q_{\gamma(\alpha \oplus \beta) y z}^{i}$, such that

$$
E_{\alpha}^{\cdot x}=\sum_{i=0}^{2} q_{\alpha x}^{i} E_{i}, \quad E_{\alpha \beta}^{\cdot y z}=\sum_{i=0}^{2} q_{\alpha \beta y z}^{i} E_{i}
$$

We call the parameters $q_{\alpha x}^{i}$ and $q_{\alpha \beta y z}^{i}$ defined in (13) the generalized Krein parameters of the strongly regular graph $X$. Notice that $q_{\alpha 2}^{i}$ and $q_{\alpha \beta 11}^{i}$ are precisely the Krein parameters of $X$ already presented. With this notation, the Greek letters are used as idempotent indices and the Latin letters are used as exponents of Hadamard (Kronecker) powers.

\section{Relations between the Krein Parameters and the Generalized Krein Parameters}

In this section we prove that the generalized Krein parameters can be expressed in function of the Krein parameters. Before that, it is worth to mention that the previously introduced generalizations are straightforward extended to the Krein parameters of symmetric association schemes with $d(d \geq 3)$ classes, see [9]. Notice that the algebra spanned by the matrices of a symmetric association scheme with $d$ classes is an Euclidean Jordan Algebra with rank $d+1$ and with the Jordan product $A \circ B=\frac{A B+B A}{2}$ where $A B$ is the usual product of matrices. Furthermore, the inner product of $\mathcal{V}$ is defined as $\langle A, B\rangle=\operatorname{tr}(A B)$ where $\operatorname{tr}($.$) is the classical$ trace of matrices, that is, the sum of its eigenvalues. Let us consider the matrices $P$ and $Q$ of the Bose-Mesner algebra of an association scheme with $d$ classes as defined in [14]. However, for convenience, we denote this matrix $Q$ such as defined in [14] by $Q^{*}$. Therefore, we can say that $Q=\frac{1}{n} Q^{*}$, see [14]. Hence, we can say that the matrices $P$ and $Q$ satisfy,

$$
\begin{gathered}
Q_{i j} Q_{i k}=\sum_{l=0}^{2} q_{j k}^{l} Q_{i l} \\
\left|Q_{i j}\right| \leq \frac{\mu_{j}}{n} \\
\left|P_{i j}\right| \leq n_{j}
\end{gathered}
$$




$$
\sum_{i=0}^{2} n_{i} Q_{i j} Q_{i k} \leq \frac{u_{j}}{n} \delta(j, k)
$$

The matrices $P$ and $Q$ are usually called the eigenmatrix and the dual eigenmatrix of the association scheme, respectively.

Theorem 1. Let $G$ be a $(n, k, a, c)$-strongly regular graph such that $0<c \leq k<n-1$ whose adjacency matrix is $A$ and has the eigenvalues $k, \theta$ and $\tau$ and whose eigenmatrix and dual eigenmatrix matrix are respectively $P$ and $Q$ If $j, k$ and $l$ are natural numbers such that $0<j, k, l \leq 2$, then

$$
q_{j k 11}^{l}=\sum_{i=0}^{2} Q_{i j} Q_{i k} P_{l i} .
$$

Proof. Consider that $\left\{E_{0}, E_{1}, E_{2}\right\}$ is the of idempotents defined in (12) and the following notation $A_{0}=I_{n}$, $A_{1}=A$ and $A_{2}=J_{n}-A-I_{n}$.

For $j, k, l \in\{0,1,2\}$ since $E_{j}=\sum_{i=0}^{2} Q_{i j} A_{i}$ and $E_{k}=\sum_{i=0}^{2} Q_{i k} A_{i}$, it follows that $E_{j} \cdot E_{k}=\sum_{i=0}^{2} Q_{i j} Q_{i k} A_{i}$. Therefore $E_{j} \cdot E_{k} E_{l}=\sum_{i=0}^{2} Q_{i j} Q_{i k} A_{i} E_{l}$ and since $A_{j}=\sum_{t=0}^{2} P_{t j} E_{t}$ implies $A_{i} E_{l}=P_{l i} E_{l}$ we obtain

$$
E_{j} \cdot E_{k} E_{l}=\sum_{i=0}^{2} Q_{i j} Q_{i k} P_{l i} E_{l} .
$$

Finally, from (19) the result follows.

Theorem 2. Let $G$ be a $(n, k, a, c)$-strongly regular graph such that $0<c \leq k<n-1$ whose adjacency matrix is $A$ and has the eigenvalues $k, \theta$ and $\tau$ and whose eigenmatrix and dual eigenmatrix matrix are respectively $P$ and $Q$. Let $j, m$ and $s$ be natural numbers such that $0 \leq j, s \leq 2$. Then

$$
q_{j m}^{s}=\sum_{i=0}^{2}\left(Q_{i j}\right)^{m} P_{s i} .
$$

Proof. Taking into account that $Q_{j}^{\cdot m}=\sum_{i=0}^{2} q_{j m}^{i} E_{i}$ and by the equalities (21) and (22)

$$
\begin{gathered}
E_{j}=\sum_{i=0}^{2} Q_{i j} A_{i}, \\
A_{i}=\sum_{j=0}^{2} P_{j i} E_{j},
\end{gathered}
$$

we conclude that $E_{j}^{\cdot m}=\sum_{i=0}^{2}\left(Q_{i j}\right)^{m} A_{i}$ Therefore $q_{j m}^{s} E_{s}=E_{j}^{\cdot m} E_{s}=\sum_{i=0}^{2}\left(Q_{i j}\right)^{m} A_{i} E_{s}$ and since by (22) $A_{i} E_{s}=P_{s i} E_{s}$ we obtain $q_{j m}^{s} E_{s}=\sum_{i=0}^{2}\left(Q_{i j}\right)^{m} P_{s i} E_{s}$. Hence $q_{j m}^{s}=\sum_{i=0}^{2}\left(Q_{i j}\right)^{m} P_{s i}$.

As an application of the Theorem 2 we may conclude that considering a strongly regular graph $G$ the generalized Krein parameters $q_{j m}^{l}$ can be expressed in function of the classical Krein parameters as follows:

$$
q_{j m}^{s}=\sum_{l_{1}=0 l_{2}=0}^{2} \sum_{l_{m-2}=0}^{2} q_{j 2}^{l_{1}} q_{l_{1} j 11}^{l_{2}} \cdots q_{(m-3) j 11}^{l_{m-2}} q_{l_{m-2} j 11}^{s} .
$$

The expression (23) is obtained using (14) and (20). Summarizing, we have the following corollary.

Corollary 1. Let $G$ be a $(n, k, a, c)$-strongly regular graph such that $0<c \leq k<n-1$. Then for all natural numbers $j, m$ and $s$ such that $0 \leq j, s \leq 2$

$$
q_{j(2+m)}^{s}=\sum_{l_{1}=0 l_{2}=0}^{2} \sum^{2} \cdots \sum_{l_{m}=0}^{2} q_{j 2}^{l_{1}} q_{l_{1 j} j 1}^{l_{2}} \cdots q_{l_{m-1} j 11}^{l_{m}} q_{l_{m} j 11}^{s} .
$$

Theorem 3. Let $G$ be a $(n, k, a, c)$-strongly regular graph such that $0<c \leq k<n-1$. Then for all natural numbers $i, j, m, n$ and $s$ such that $0 \leq i, j, s \leq 2$,

$$
q_{i j m n}^{s}=\sum_{l=0}^{2}\left(Q_{l i}\right)^{m}\left(Q_{l j}\right)^{n} P_{s l} .
$$


Proof. We have $E_{i}^{\cdot m} \cdot E_{j}^{\cdot n}=\sum_{l=0}^{2} q_{i j m n}^{l} E_{l}$. Since from (21) $E_{i}^{\cdot n} \cdot E_{j}^{m}=\sum_{l=0}^{2}\left(Q_{l i}\right)^{n} A_{1} \cdot \sum_{l=0}^{2}\left(Q_{l i}\right)^{m} A_{l}$ then $E_{i}^{\cdot n} \cdot E_{j}^{\cdot m}=\sum_{l=0}^{2} q_{i j m n}^{l} E_{l}$. Hence we obtain

$$
E_{i}^{\cdot n} \cdot E_{j}^{m} E_{s}=\sum_{l=0}^{2}\left(Q_{l i}\right)^{n}\left(Q_{l j}\right)^{m} P_{s l} E_{s} .
$$

Therefore, the equality (25) follows.

Recurring to (14) and (25), we may conclude the Corollary 2.

Corollary 2. Let $G$ be a $(n, k, a, c)$-strongly regular graph such that $0<c \leq k<n-1$. Then for all natural numbers $i_{1}, i_{2}, m, n$ and $s$ such that $0 \leq i_{1}, i_{2}, s \leq 2$,

$$
q_{i_{1} i_{2} n m}^{s}=\sum_{l_{1}=0}^{2} \cdots \sum_{l_{n}=0 l_{n+1}=0}^{2} \sum_{l_{n+m-2}=0}^{2} q_{i_{1} 2}^{l_{1}} q_{l_{1} i_{11}}^{l_{2}} \cdots q_{l_{n-2} i_{1} 11}^{l_{n-1}} q_{l_{n-1} i_{2} 11}^{l_{n}} q_{l_{n_{2} i_{1} 11}}^{l_{n+1}} \cdots q_{l_{n+m-3} i_{2} 11}^{l_{n+m-2}} q_{l_{n+m-2} i_{2} 11}^{s} .
$$

Theorem 4. Let $G$ be a $(n, k, a, c)$-strongly regular graph such that $0<c \leq k<n-1$. Then $\forall n \in \mathbb{N}$ and $\forall i_{1}, \cdots, i_{n+1} \in\{0,1,2\}$,

$$
\sum_{r=0}^{2} Q_{r_{1}} \cdots Q_{r_{n+1}} P_{s r} \geq 0
$$

Proof. We prove by induction on $n$. For $n=1$ the inequality (26) holds, since the classical Krein parameters $q_{i_{1} i_{2} 11}^{s}$ are nonnegative and $\sum_{r=0}^{2} Q_{r i_{1}} Q_{r i_{2}} P_{s r}=q_{i_{1} i_{2} 11}^{s}$. Now assuming that the inequality (26) holds for $n=k \geq 1$, we prove that (26) also holds for $n=k+1$. Consider the sum $\sum_{r=0}^{2} Q_{r_{1}} \cdots Q_{r_{k+2}} P_{s r}$. Then from (14), we obtain

$$
\begin{aligned}
\sum_{r=0}^{2} Q_{r_{1}} \cdots Q_{r i_{k+2}} P_{s r} & =\sum_{r=0 l=0}^{2} \sum^{2} q_{i_{1} i_{2} 11}^{l} Q_{r l} Q_{r_{i}} \cdots Q_{r_{k+2}} P_{s r} \\
& =\sum_{l=0}^{2} q_{i_{i} 1211}^{l} \sum_{r=0}^{2} Q_{r l} Q_{r_{i}} \cdots Q_{r_{k+2}} P_{s r} .
\end{aligned}
$$

Since for $n=k$ the inequality (26) is verified and the summands $\sum_{r=0}^{2} Q_{r l} Q_{r i_{3}} \cdots Q_{r_{k+2}} P_{s r}$ are nonnegative, we may conclude that $\sum_{r=0}^{2} Q_{r_{1}} \cdots Q_{r_{n+1}} P_{s r} \geq 0$.

Recurring to the Theorem 4 we are conducted to the Corollaries 3 and 4.

Corollary 3. Let $G$ be a $(n, k, a, c)$-strongly regular graph such that $0<c \leq k<n-1$. Then for all natural numbers $i, m, s$ and $s$ such that $0 \leq i, s \leq 2$ the generalized Krein parameters $q_{i m}^{s}$ are nonnegative.

Corollary 4. Let $G$ be a $(n, k, a, c)$-strongly regular graph such that $0<c \leq k<n-1$. Then for all natural numbers $i, j, m, n$ and such that $0 \leq i, j, s \leq 2$ the generalized Krein parameters $q_{i j m n}^{l}$ are nonnegative.

Theorem 5. Let $G$ be a strongly regular graph and let $i, s$ and $m$ be natural numbers such that $0 \leq i, s \leq 2$. Then $q_{i m}^{s} \leq 1$.

Proof. Recurring to the inequalities (14)-(17) we have:

$$
\begin{aligned}
q_{i m}^{s} & =\sum_{t=0}^{2}(Q(t, i))^{m} P(s, t)=\left|\sum_{t=0}^{2}(Q(t, i))^{m-2}(Q(t, i))^{2} P(s, t)\right| \\
& \leq \sum_{t=0}^{2}\left|(Q(t, i))^{m-2}\right|\left|(Q(t, i))^{2}\right||P(s, t)| \leq \sum_{t=0}^{2}\left(\frac{\mu_{i}}{n}\right)^{m-2}(Q(t, i))^{2} n_{t} \\
& \leq \sum_{t=0}^{2} 1^{m-2}(Q(t, i))^{2} n_{t} \leq \frac{\mu_{i}}{n} \leq 1 .
\end{aligned}
$$

Theorem 6. Let $G$ be a $(n, k, a, c)$-strongly regular graph such that $0<c \leq k<n-1$. Let $i, j, m, n$ and $s$ be natural numbers such that $0 \leq i, j, s \leq 2$ and $m+n \geq 3$. Then the generalized Krein parameter $q_{i j m n}^{s}$ satisfy $q_{i j m n}^{s} \leq 1$.

Proof. Similar to the Proof done in Theorem 5.

Let $G$ be a $(n, k, a, c)$-strongly regular graph such that $0<c \leq k<n-1$. Since the generalized Krein parameters $q_{i, i m n}^{s}$ and $q_{i, m}^{s}$ are nonnegative then we can establish new admissibility conditions distinct from the Krein conditions (6) and (7). For instance, the generalized Krein condition $q_{13}^{0} \geq 0$ allows us to establish a new 
theorem on strongly regular graphs after some algebraic manipulation of its expressions. Analyzing the generalized Krein parameter $q_{13}^{0}$ of a strongly regular graph with one in its spectra we deduce the following theorem (7).

Theorem 7. Let $G$ be a $(n, k, a, c)$-strongly regular graph such that $0<c \leq k<n-1$ whose adjacency matrix is $A$ and has the eigenvalues $k, \theta=1$ and $\tau$ If $k \geq 9$ then

$$
n \leq \frac{3}{2}+\frac{53}{20} k
$$

Proof. Since $q_{13}^{0} \geq 0$ then we have

$$
\left(\frac{\theta n+k-\theta}{n(\theta-\tau)}\right)^{3}+\left(\frac{-n+k-\theta}{n(\theta-\tau)}\right)^{3} k+\left(\frac{k-\theta}{n(\theta-\tau)}\right)^{3}(n-k-1) \geq 0 .
$$

From the inequality (28) and after some simplifications we conclude that

$$
n^{2}\left(\theta^{3}-k\right)+n\left(-3 \theta^{3}+3 k^{2}+3 \theta^{2} k-3 k \theta\right)+\left(2 \theta^{3}-2 k^{3}+6 \theta k^{2}-6 \theta^{2} k\right) \geq 0 .
$$

Therefore if $\theta=1$ then

$$
n^{2}(1-k)+n\left(-3+3 k^{2}+3 k-3 k\right)+\left(2-2 k^{3}+6 k^{2}-6 k\right) \geq 0
$$

Finally we have

$$
n^{2}(1-k)+n\left(-3+3 k^{2}\right)+2\left(1-k^{3}+3 k^{2}-3 k\right) \geq 0
$$

Dividing both members of (29) by $1-k$ we are supposing that $k>1$ we obtain

$$
n^{2}-3 n(1+k)+2\left(k^{2}-2 k+1\right) \leq 0 .
$$

Now from the inequality (30) we conclude that if $G$ is a $(n, p, a, c)$-strongly regular graph with one in his spectra $^{1}$ then $k \geq 9$ implies that $n \leq \frac{3}{2}+\frac{53}{20} k$.

We now present in Table 1 some examples of parameter sets $(n, k, a, c)$ that do not verify the inequality (27) of Theorem 7. We consider the parameter sets $P_{1}=(28,9,0,4), P_{2}=(64,21,0,10), P_{3}=(1225,456,39,247)$, $P_{4}=(1296,481.0,40.0,260)$ and $P_{5}=(1024,385,36,210)$. For each example we present the respective eigenvalues $\theta, \tau$ and the value of $q_{k n}$ defined by $q_{k n}=\frac{53}{20} k+\frac{3}{2}-n$.

\section{Some Conclusions}

In this paper, we have generalized the Krein parameters of a strongly regular graph and obtained some relations

Table 1. Numerical results when $k \geq 9$.

\begin{tabular}{cccccc}
\hline & $P_{1}$ & $P_{2}$ & $P_{3}$ & $P_{4}$ & $P_{5}$ \\
\hline$\theta$ & 1 & 1 & 1 & 1 & 1 \\
$\tau$ & -5 & -11 & -209 & -221 & -175 \\
$q_{\theta r k n}^{1}$ & -2.65 & -6.85 & -15.1 & -19.85 & -2.5 \\
\hline
\end{tabular}

${ }^{1}$ We must note that the equation $x^{2}-3 x(1+k)+2\left(1-2 k+k^{2}\right)=0$ as the roots $x_{1}=\frac{3(k+1)-\sqrt{k^{2}+34 k+1}}{2}$ and the root $x_{2}=\frac{3(k+1)+\sqrt{k^{2}+34 k+1}}{2}$. Since $k>8$ implies that $k^{2}+34 k+1 \leq 5 k^{2}$ and finally this implies that $x_{2} \leq \frac{3(k+1)+\sqrt{5} k}{2}$ therefore $x_{2} \leq \frac{3}{2}+\frac{53 k}{20}$. 
between the classical Krein parameters and the generalized Krein parameters (see Corollaries 1 and 2). We also establish that these generalize Krein parameters are always positive and less than one (see Corollaries 3 and 4, and Theorems 5 and 6). Let $i, j, m, n$ and $s$ be natural numbers such that $0 \leq i, j, s \leq 2$. The generalized Krein admissibility conditions $q_{i j m n}^{s} \geq 0$ with $m+n \geq 3$ and $q_{i m}^{s} \geq 0$ with $m \geq 3$ allow us to establish new admissibility conditions; they permit us to establish new inequalities on the parameters of a strongly regular graph. For instance the generalized Krein parameter condition $q_{23}^{0} \geq 0$ after some algebraic manipulation allows us to establish the inequality (27) in Theorem 7. Finally, we conclude that we can extend the definition of generalized Krein parameters to a symmetric association scheme with $d$ classes.

\section{Acknowledgements}

1) Luís Almeida Vieira was supported by the European Regional Development Fund through the program COMPETE and by the Portuguese Government through the FCT—Fundação para a Ciência e a Tecnologia under the project PEst-C/MAT/UI0144/2013.

2) Vasco Moço Mano was partially supported by Portuguese Funds trough CIDMA-Center for Research and development in Mathematics and Applications, Department of Mathematics, University of Aveiro, 3810-193, Aveiro, Portugal and the Portuguese Foundation for Science and Technology (FCT-Fundação para a Ciência e Tecnologia), within Project PEst-OE/MAT/UI4106/2014.

3) The authors would like to thank the anonymous referee for his/her careful revision and relevant comments that improved our paper.

\section{References}

[1] McCrimmon, K. (1978) Jordan Algebras and Their Applications. Bulletin of the American Mathematical Society, 84, 612-627. http://dx.doi.org/10.1090/S0002-9904-1978-14503-0

[2] Jordan, P., Neuman, J.V. and Wigner, E. (1934) On an Algebraic Generalization of the Quantum Mechanical Formalism. Annals of Mathematics, 35, 29-64. http://dx.doi.org/10.2307/1968117

[3] Massan, H. and Neher, E. (1998) Estimation and Testing for Lattice Conditional Independence Models on Euclidean Jordan Algebras. Annals of Statistics, 26, 1051-1082. http://dx.doi.org/10.1214/aos/1024691088

[4] Faybusovich, L. (1997) Euclidean Jordan Algebras and Interior-Point Algorithms. Positivity, 1, 331-357. http://dx.doi.org/10.1023/A:1009701824047

[5] Faybusovich, L. (2007) Linear Systems in Jordan Algebras and Primal-Dual Interior-Point Algorithms. Journal of Computational and Applied Mathematics, 86, 149-175. http://dx.doi.org/10.1016/S0377-0427(97)00153-2

[6] Cardoso, D.M. and Vieira, L.A. (2004) Euclidean Jordan Algebras with Strongly Regular Graphs. Journal of Mathematical Sciences, 120, 881-894. http://dx.doi.org/10.1023/B:JOTH.0000013553.99598.cb

[7] Koecher, M. (1999) The Minnesota Notes on Jordan Algebras and Their Applications. Krieg, A. and Walcher, S., Eds., Springer, Berlin.

[8] Faraut, J. and Korányi, A. (1994) Analysis on Symmetric Cones. Oxford Science Publications, Oxford.

[9] Godsil, C. and Royle, G. (2001) Algebraic Graph Theory. Springer, Berlin. http://dx.doi.org/10.1007/978-1-4613-0163-9

[10] van Lint, J.H. and Wilson, R.M. (2004) A Course in Combinatorics. Cambridge University Press, Cambridge.

[11] Scott Jr., L.L. (1973) A Condition on Higman’s Parameters. Notices of the American Mathematical Society, 20 , A-97.

[12] Delsarte, Ph., Goethals, J.M. and Seidel, J.J. (1975) Bounds for Systems of Lines and Jacobi Polynomials. Philips Research Reports, 30, 91-105.

[13] Bose, R.C. and Mesner, D.M. (1952) On Linear Associative Algebras Corresponding to Association Schemes of Partially Balanced Designs. The Annals of Mathematical Statistics, 47, 151-184.

[14] Brower, A.E. and Haemers, W.H. (1995) Association Schemes. In: Grahm, R., Grotsel, M. and Lovász. L., Eds., Handbook of Combinatorics, Elsevier, Amsterdam, 745-771. 
Scientific Research Publishing (SCIRP) is one of the largest Open Access journal publishers. It is currently publishing more than 200 open access, online, peer-reviewed journals covering a wide range of academic disciplines. SCIRP serves the worldwide academic communities and contributes to the progress and application of science with its publication.

Other selected journals from SCIRP are listed as below. Submit your manuscript to us via either submit@scirp.org or Online Submission Portal.
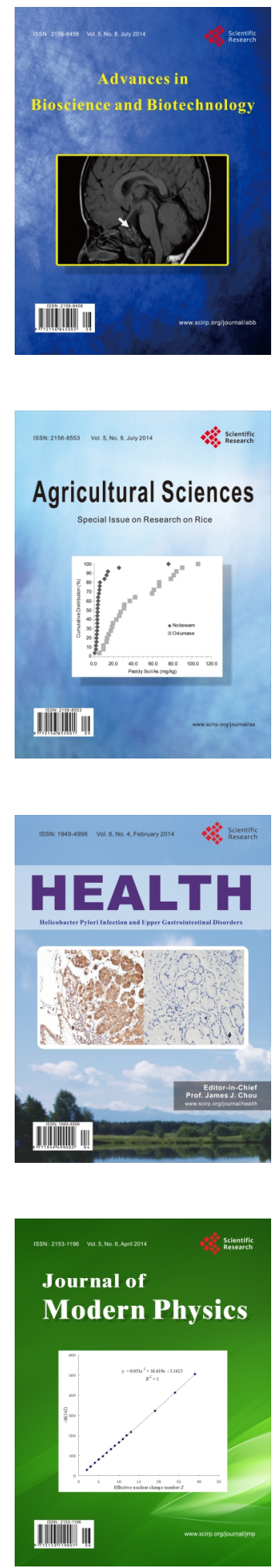
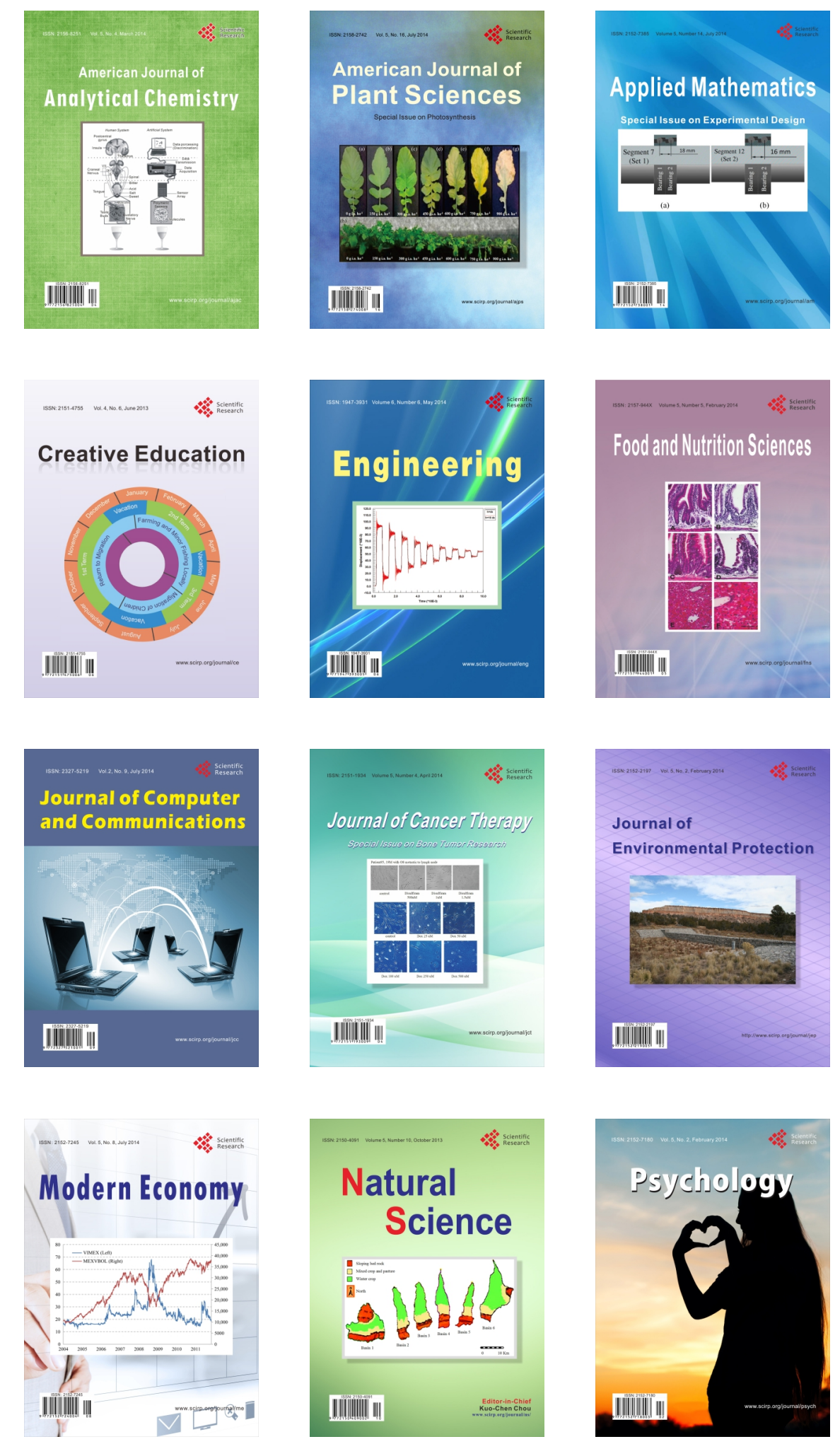